occurred in BDI litigation cases was investigated by de Reuver et al. In this Dutch study, 13 independent experts reviewed $10 \mathrm{BDI}$ litigation cases that were selected to provide a distribution of injury type and disease-related mortality. The 13 experts unanimously agreed that negligence had occurred in only 1 of the 10 cases. In seven cases, a maximum agreement of just $53 \%$ was reached, indicating that roughly half of the experts judged that negligence had occurred, whereas the others judged the opposite, or could not determine whether negligence had occurred.

Of note, some experts were consistently severe in their judgment of negligence, while others were consistently mild. Judgments of negligence were associated with BDI-related mortality, but not with clinical characteristics of the BDI or with delayed diagnosis.

The authors noted that most experts strongly recommended the assignment of more than one expert witness to testify in surgical litigation cases. This study reveals the poor agreement among surgical experts in the assessment of negligence in malpractice litigation, and suggests that guidelines for surgical-witness testimony must be developed to improve agreement in judgments on negligence.

Original article de Reuver PR et al. (2008) Poor agreement among expert witnesses in bile duct injury malpractice litigation: an expert panel survey. Ann Surg 248: 815-820

\section{Economics of Helicobacter pylori eradication in clinical practice}

The cost of PPI therapy in primary care is considerable, and the number of PPI prescriptions is increasing. The main indications for PPI therapy are gastrointestinal-related and include dyspepsia, GERD and gastroprotection. The eradication of Helicobacter pylori is probably beneficial for a percentage of patients on long-term PPI therapy who are infected with it, as successful eradication could lead to a reduction in PPI use; however, the use of a 'test and treat' strategy for $\mathrm{H}$. pylori infection is not currently standard clinical practice.

Mason and colleagues performed an economic analysis of the multicenter UK HELPUP trial to establish whether the eradication of $H$. pylori is cost-effective in primary-care patients on long-term PPI therapy who have a positive result for $H$. pylori infection in an urea breath test (UBT). In total, 183 patients were randomly allocated to receive eradication therapy or placebo for 1 week. At 2-year follow-up, numbers of PPI prescriptions, physician consultations, upper gastrointestinal endoscopies, home visits related to gastrointestinal complaints, and abdominal ultrasound scans were all significantly reduced in patients who received eradication therapy compared with those who received placebo. Average net savings per patient were UK£93 (95\% Cl33-153). Symptoms of dyspepsia and quality of life were also significantly improved in patients who received eradication therapy.

A second study, performed by Vakil and colleagues, examined the cost and accuracy of the standard practice of performing a duplicate UBT (4 weeks after the first) to confirm eradication of $H$. pylori infection after therapy. The analysis included 419 patients with $H$. pylori infection (documented by a positive UBT and histology) who had taken part in prospective, randomized controlled studies of $H$. pylori eradication. Patients received eradication therapy with one of three therapeutic regimens followed by UBTs 4 weeks and 8 weeks after treatment. No discordant results were reported between the first and second UBTs in all patients. The additional cost of performing a second UBT was high $(€ 12,570$ in this study; in the US this cost would be $\$ 46,090$ ) and did not contribute any incremental clinical benefit. The one-test strategy was significantly less expensive than the two-test strategy. The authors concluded that "... a single UBT, 4 weeks after treatment is as effective as two serial breath tests in confirming $H$. pylori eradication."

The studies by these two research groups indicate the potential economic effects of performing a 'test and treat' strategy in primarycare, long-term PPI users, and performing a single UBT to confirm $H$. pylori eradication after therapy. These two strategies could significantly reduce overall health-care costs and the severity of symptoms associated with $H$. pylori infection.

Original articles Mason JM et al. (2008) Helicobacter pylori eradication in long-term proton pump inhibitor users is highly cost-effective: economic analysis of the HELPUP trial. Aliment Pharmacol Ther 28: 1297-1303

Vakil N et al. (2008) Duplicate breath testing to confirm eradication of Helicobacter pylori: incremental benefit and cost in 419 patients. Aliment Pharmacol Ther 28: 1304-1308 